\title{
Learning in Computer Vision: Some Thoughts
}

\author{
Maria Petrou \\ Communications and Signal Processing Group, \\ Electrical and Electronic Engineering Department, \\ Imperial College, \\ London SW7 2AZ, UK
}

\begin{abstract}
It is argued that the ability to generalise is the most important characteristic of learning and that generalisation may be achieved only if pattern recognition systems learn the rules of meta-knowledge rather than the labels of objects. A structure, called "tower of knowledge", according to which knowledge may be organised, is proposed. A scheme of interpreting scenes using the tower of knowledge and aspects of utility theory is also proposed. Finally, it is argued that globally consistent solutions of labellings are neither possible, nor desirable for an artificial cognitive system.
\end{abstract}

\section{Introduction}

The word "learning" has many interpretations among the pattern recognition community in general, and the computer vision community in particular. It has been used to loosely mean anything between the identification of the best value of a parameter from training data, to learning how to recognise visual structures. So, perhaps we should try to distinguish the interpretation of the word as it appears in the mathematical formulation of problems, from its interpretation as it appears in cognition related tasks. In the sections that follow, we shall try to understand the significance of learning in the context of computer vision and identify a possible relationship between these two interpretations of the word.

\section{The Main Characteristic of Learning}

Some of the first learning algorithms in relation to cognitive tasks, that have been proposed in the past, are the various types of neural network. Proponents of neural networks often comment on the generalisation capabilities of the networks they develop. Generalisation is one of the characteristics of learning. Indeed, we, humans, teach our children often with fairy tales and parables, assuming that they have the ability to generalise to real situations. It is preposterous to expect that we shall have to teach our children about all individual possible situations they may encounter in life, in order to make sure that we have taught them well. We may safely conclude, therefore, that the ability to generalise is the most important characteristic of learning. 
This implies that classical pattern recognition methods, that use training examples to span the feature space, are not really learning methods in the cognitive sense of the word. Even neural network based methods, in order to generalise well, rely heavily on the availability of enough training samples to populate adequately the feature space. The training patterns are used by the neural networks to approximate the class boundaries in the feature space with piece-wise linear segments. When an unknown pattern arrives, it can be associated with the class that has been identified to populate the part of the feature space where the pattern appears. Some old [3] and some more recently developed methods [1], that can work with fewer training patterns than straightforward methods, do so by selecting the patterns that matter most in defining the class boundaries, rather than by using some higher level generalisation abilities of the classifier [23]. So, neural networks and pattern classification methods are not learning methods in the cognitive sense of the word.

The question then that arises is: is learning, as understood by cognitive scientists and psychologists, algorithmic, or is it something beyond the current grasp of mathematical formalism? Evidence against learning being algorithmic is the ability of humans to learn even from single examples. A counter-argument is that humans take a lot of time to learn, and it is possible that what we witness as super-fast learning is simply a manifestation of the application of some meta-knowledge, some generic rules that have been slowly and painfully learnt subconsciously. I would like to clarify that "learning" here does not refer to getting a University degree. This is indeed a very long process and it takes almost one third of the life span of a person. Learning here refers to survival skills, to skills needed to operate in everyday life. Given that a human becomes reproductive roughly between the ages of $12-15$, we may assume that nature considers the human child becoming ready for life at that stage. So, we may say that humans "learn" what they have to learn, to be ready for life as independent entities, over a period of 12-15 years, which is still a significant fraction of their life span. Therefore, humans seem to be slow learners after all. They take a lot of time to work out the rules of meta-knowledge. It is these rules, that have possibly been learnt in an algorithmic way, that allow then the human to learn in the super-fast, almost magical, way that we often witness. We may conclude from the above arguments that:

- generalisation is an important characteristic of learning;

- generalisation in algorithmic learning may only be achieved by having enough training examples to populate all parts of the class space, or at least the parts that form the borders between classes;

- we have true generalisation capabilities, only when what is learnt by training examples are rules on how to extract the identity of objects and not the classes of objects directly. If such learning has taken place, totally unknown objects may be interpreted correctly, even in the absence of any previously seen examples.

This conclusion implies that what we have to teach the computer, in order to construct a cognitive system, are relations rather than facts. For example, memorising 
the dictionary of a language, does not teach a person the language. The person has to learn the relations between words in order to master the language. This is in agreement with Winstone's pioneering experiments on teaching the computer to recognise arches. He did not show to the computer all possible types of arch it may encounter. He showed it examples and counter examples of arches and taught it to recognise relations between components, such as "supports" or "is supported by" [26].

\section{Knowledge and Meta-knowledge}

There are at least 50 theories of learning developed by cognitive scientists 28]. In computer science, we tend to distinguish two forms of learning: learning by experimentation and learning by demonstration. Inductive learning is also used by computer scientists, but we shall not deal with that here, because it implies that the learner has already learnt the rules of logic. So, inductive learning may be thought of as a way of applying the already learnt rules of meta-knowledge 22.

Learning by experimentation implies the ability to try, reason about the results and draw conclusions. In its simplest form, this is what fully automatic programs do. A fully automatic segmentation algorithm, for example, will work as follows: perform segmentation, assess the quality of the result, adjust the parameters and try again. The immediate conclusion is that learning by experimentation requires the presence of a feed-back loop. It also requires the availability of a criterion that says how well the system has performed each time. It is important for this criterion to be independent of the knowledge or information used to perform the segmentation in the first place, otherwise the result will be a self-fulfilling prophecy. In semi-automatic systems, the criterion is provided by the human. The reported results in the literature then include a statement of the type: "the best threshold was found by trial and error." This method is a perfectly legitimate method of learning. It comes under the umbrella of supervised learning and it corresponds to human learning with the help of a teacher.

Proceeding to fully automated methods is equivalent to assuming that the computer has somehow passed that stage of learning the meta-knowledge, necessary to construct rules, and now learning proceeds very fast, making use of these meta-rules. For example, if we know that agricultural fields tend to have straight borders, we may judge the output of a segmenter of an image, captured by an airborne sensor, as good or bad, according to whether it produced regions with straight borders or not. The knowledge that fields have straight boundaries is a meta-knowledge. The segmenter might have operated using only texture and edge information. The straightness of the inferred borders may be used as a criterion to drive the system to use its feed-back loop to work out a better segmentation. The question then is: how did the system acquire this meta-knowledge? As argued earlier, it must be the meta-knowledge that had to be learnt by the human child (or the computer learner) painfully slowly by seeing lots of examples of agricultural fields. And although no method has been found yet to transplant this meta-knowledge to the brain of the human child 
from the brain of the teacher, computers have an advantage here: the teacher, i.e. the human, may insert the meta-knowledge into the system while developing the criterion of self assessment of the algorithm. From this line of argument, we conclude that:

- meta-knowledge may take the form not only of relations, but also of generic characteristics that categories of objects have;

- in interactive systems, meta-knowledge is inserted into the computer learner by the human teacher manually;

- in automatic systems, meta-knowledge is supplied to the computer learner by the human teacher in the form of a criterion of performance assessment.

Two questions then arise:

- what connects the knowledge with the meta-knowledge?

- how is meta-knowledge learnt in the first place?

\section{Learning by Demonstration}

To answer the above questions, we get a clue from the second type of learning we mentioned earlier, namely learning by demonstration. The demonstrator here is the teacher. The next is a story I heard from my grandmother. Remember that the traditional way of teaching children has always been through stories and parables. This story offers the clue we are searching for.

'Once upon a time there was a potter who got an apprentice who wanted to learn the art of pottery. The potter made his clay pots and put them in the oven. After two hours, he turned the fire off, and sat down to rest and smoke, as he was an old man. Then he took the pots out of the oven. They were perfect. The apprentice later decided to do his own pots. He made them out of clay and put them in the oven. After two hours, he took them out. The pots broke. He repeated the task and he had the same results. He went back to the potter: "You did not teach me well. Such and such happened." "Did you stop to smoke after you switched off the fire?" "No, I am not a smoker." "So, you got the pots out of the oven too soon.",

I am sure the story was related to me in order to teach me to pay attention to the detail. Indeed, if the apprentice had seen the potter performing the act dozens of times with slight variation each time, but always with the pause before the pots were taken out of the oven, he might have worked out that that pose was crucial to the process. On the other hand, the teacher might have been a better teacher if he had made that information explicit.

So, this story tells us that we learn fast, from very few examples, only when somebody explains to us why things are done the way they are done. A child asks lots of "why"s and that is how a child learns. This tells me that we cannot disassociate learning to recognise objects from learning why each object is the way it is. One may consider the following exchange between a teacher and a learner: 
"What is this?"

"This is a window."

"Why?"

"Because it lets the light in and allows the people to look out."

"How?"

"By having an opening at eye level."

"Does it really?"

This sequence of learning is shown in Fig. 1. This figure proposes that knowledge in our brain is represented by a series of networks, forming a complex structure that I call the "tower of knowledge". The network of nouns is a network of object names, labels, e.g. "window", "chimney", "door", etc. The network of verbs or actions, is a network of functionalities, e.g. "to look out", "to enter", "to exit", etc. The network of appearances is a network of basic shapes necessary for a functionality to be fulfilled, e.g. "it is an opening of human size at floor level". So, the flow of knowledge goes like the fragment of conversation given above. The loop closes when we confirm that the object we are looking at has the right characteristics for its functional purpose to be fulfilled.

The task, therefore, for the artificial vision scientist, is to model these layers of networks and their inter-connections. We have various tools at our disposal: Markov Random Fields 8], grammars [19], inference rules [24], Bayesian networks [16, Fuzzy inference 27, etc. I would exclude from the beginning any deterministic crisp approaches, either because things are genuinely random in nature (or at least have a significant random component), or because our models and our knowledge is far too gross and imperfect for creating crisp rules and dogmatic decisions.

\section{Markov Random Fields}

Some recent work [17] showed evidence that the network of nouns (better described in psychophysical terms as network of "ideas") is topologically a random network, while the network of relations, made up from pairs of ideas, is topologically scale-free. For example, pairs like "fork-knife", "door-window" come up much more frequently in trains of thought than "door" alone, or "window" alone. This indicates that the connections in these networks are of varied strength, and actually are not always symmetric. For example, the idea "door" may trigger the idea "window" more frequently than the idea "window" triggers the idea "door". This asymmetry in the interactions is a manifestation that Markov Random Fields (MRFs) are not applicable here in their usual form in which they are applied in image processing. An example of the interactions in a neighbourhood of an MRF, defined on a grid, is shown in Fig. 2b. This MRF, and the weights it gives for neighbouring interactions, cannot be expressed by a Gibbs joint probability density function. For example, the cell at the centre is influenced by its top left neighbour with weight -1 , but itself, being the bottom right neighbour of the cell at the top left, influences it with weight +1 . This asymmetry leads to 
instability when one tries to relax such a random field, because local patterns created are not globally consistent (and therefore not expressible by global Gibbs distributions) [18]. According to Li [91011], relaxations of such MRFs do not converge, but oscillate between several possible states. (Optimisations of Gibbs distributions either converge to the right interpretation, but more often than not, they hallucinate, i.e. they settle on wrong interpretations.)

So, one could model the network at each level of the tower of knowledge shown in Fig. 1, using a non-Gibbsian MRF [5]. The interdependences between layers might also be modelled by such networks, but perhaps it is more appropriate to use Bayesian models, as the inter-layer dependencies are causal or diagnostic, rather than peer-to-peer.

The question that arises then is: "where are we going to get the knowledge to construct these networks?" Where does the mother that teaches her child get it from? There is no "ground truth" or universal knowledge the mother transfers to her child: she sees something and talks about it to the child, then she remembers something else, according to her own network of related ideas that invoke each other and are prompted by her own sensory input, talks again to the child, and so on. So, all the mother (the teacher) does is to transfer to the child her own connections between ideas and concepts. If the mother tells the child "This is a pencil and that is a rubber. The pencil helps us write and the rubber helps us erase what we wrote.", the child will make the same connections as the mother had in her own brain. Pencil-rubber will have a strong mutual recall in the child's network of nouns, as well as write-erase in the child's network of verbs. So, one thing we can do is to model our own mental connections between ideas and functionalities. Then let the child (the computer) ask the right questions. For every answer, the strength of the corresponding connection is increased. We may turn these strengths into probabilities. Then a totally new scene may be shown to the computer. The child/computer must be able to use the connections it has learnt to interpret this new scene.

In practice, this is done by using manually annotated images. Heesch and Petrou [5] did exactly this to interpret outdoor scenes of buildings: they used hundreds of annotated images to learn the Markov dependencies of region configurations, defining the neighbourhood of a region to be the six regions that fulfil one of the following geometric constraints: it is above, below, to the left, to the right, it is contained by, or contains the region under consideration. An unknown scene was then labelled using a preliminary labelling performed on the basis of individual measurements made on each region, and relaxing the MRF defined on the segmented regions, using graph colourings and drawing labels for each region according to the local conditional probability of labels, conditioned on the current labels of the neighbours. No global consistency is guaranteed that way, but no global consistency exists, when the interdependencies between labels are asymmetric. We may intuitively understand this, as in an outdoor environment the long range interactions between objects are probably too weak to have a significant effect on the identity of a region. For example, if this region that belongs to this house here is a door, that region that is at the other end of the 

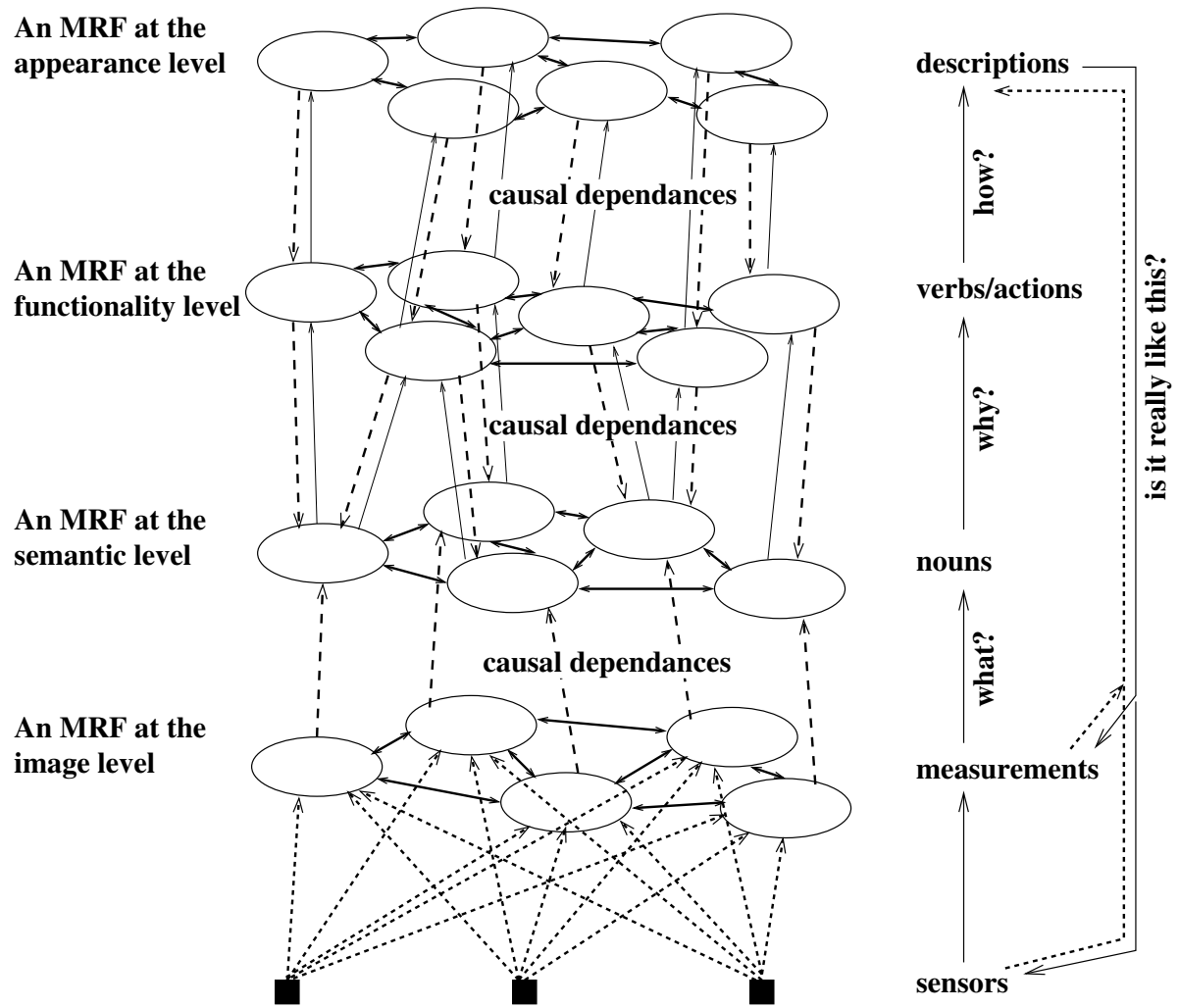

Fig. 1. The tower of knowledge: how knowledge may be organised. The double-headed arrows represent contextual interactions. The thin continuous arrows represent queries. The dashed arrows represent answers, i.e. transfer of information. The level of interest in a cognitive vision task is the level of nouns, where we wish to assign labels to objects. Examples of nodes with contextual connotations in the network of nouns are "door", "window", "balcony". Examples of nodes with contextual connotations in the network of functionality are "lets air in", "lets light in", "allows a person to enter". Examples of nodes with contextual connotations in the network of descriptions are "has a glass pane", "is at eye-level", "has a handle to open it".

field of view may be a car, a bush, a window, a house, or a tree. The differentiation of such options in terms of probabilities must be very small: the correlation function between labels, referring to objects beyond a certain distance apart, flattens out and provides no useful information. So, no global model is expected to be relevant or useful.

\section{Bayesian Inference}

Bayesian approaches have been used so far in two ways: either in the form of probabilistic relaxation (PR) [7] or in the form of Pearl-Bayes networks of 
inference [16]. Probabilistic relaxation has its origins in the seminal work on constraint propagation by Waltz [25], who used crisp constraints and solved once and for all the problem of globally inconsistent labellings that led to impossible objects 6]. Probabilistic relaxation updates the probabilities of various labels of individual objects by taking into consideration contextual information [7. As this contextual information is in effect peer-to-peer, probabilistic relaxation is not an appropriate tool for modelling causal relationships. It is rather an alternative tool to MRFs discussed in the previous section for modelling influences at the same layer. Probabilistic relaxation, just like MRF relaxation, is not guaranteed to converge to a unique global solution, unless special conditions are obeyed [21. We discussed earlier that this is not an issue in reality: labellings of scenes do not have to be globally consistent, but only locally consistent. This statement seems to be in contradiction with a previous statement, saying that probabilistic relaxation is the generalisation of Waltz's algorithm which solved the problem of inconsistent labellings in the 60s. This contradiction, however, is only superficial. The problem of inconsistent labellings of the 60s was referring to the labellings of single solid objects, by labelling their sub-parts [4] and not the labellings of scenes that contain many different objects, where constraints between objects are far weaker than constraints within the subparts of the same solid object.

The second form of Bayesian approach is that of Pearl-Bayes networks of inference. Here the relations may be causal, and so these networks are appropriate for inter-layer inference. Bayesian approaches depend on conditional probabilities. How to choose these conditional probabilities has always been a problem for such methods. Conditional probabilities may have to be learnt painfully slowly from hundreds of examples. Stassopoulou et al. 20, solved the problem of learning the conditional probabilities, by mapping the class boundaries expressed by such a network, to a neural network, which was subsequently trained to learn these conditional probabilities. Alternatively, conditional probabilities may be transferred ready from another already trained network: the network of the teacher. This transference is equivalent to choosing them to have some parametric form (e.g. Gaussian) with parameters chosen "arbitrarily". The arbitrary choice of form and parameters usually leads to the criticism of the approach being ad-hoc or unjustified. It is not, if the teacher simply transfers their own hard gained knowledge to the pupil (the computer). Such an approach leads us to new theories, like for example the so called "utility theory" 12 .

Utility theory is a decision theory. Assigning labels to objects depicted in an image is a decision. In the Bayesian framework we make this decision by maximising the likelihood of a label given all the information we have. In utility theory, this likelihood has to be ameliorated with a function called "utility function", that expresses subjective preferences or possible consequences of each label we may assign. The utility function multiplied with the Bayesian probability of each label and summed over all possibilities leads in one pass only to the final label. So, this approach avoids the iterations used by MRFs and PR. The utility function may be identified with the innate meta-knowledge somebody has acquired about the world. It is that knowledge, that might have been learnt algorithmically and 
from many examples, but which now is expressed in the form of conditions and prejudices that cannot be fully justified by the measurements we make. It is the knowledge that tells us to be cautious when we want to buy a car from a man that postponed the appointment we made several times, that did not produce immediately the maintenance record of the car we requested, and so on. Such ideas have been around for some time, without people using the term "utility function". For example, psychologists in the mid-nineties were talking about the so called pmaps and m-maps. The p-maps were meant to be the prior knowledge we have about various possible patterns that we may encounter in life. A p-map guides us to sample a scene more or less carefully at places where it matters or it does not matter, respectively, producing the m-map that is specific to the present situation. One may identify here the p-maps as being the utility functions of today and the m-maps the Bayesian part of labels conditioned on the measurements we have $\operatorname{made} 1$.

In the computer vision context, utility theory has been used by Marengoni [13 to select the features and operators that should be utilised to label aerial images. Further, one may interpret the work of Miller et al. [14 as using a utility function that penalises the unusual transformations that will have to be adopted to transform what is observed to what the computer thinks it is. The authors effectively choose labels by maximising the joint likelihood of the probability density function of the observed transforms and the probability density function of the labels and observations, assuming that transforms and labels/measurements are independent.

\section{Modelling the "Why" and the "How" in Order to Answer the "What"}

Let us consider the tower of knowledge presented in Fig. 1. We shall formulate here the problem of learning to recognise objects in a scene, using this hierarchical representation of knowledge and utility theory.

Let us assume that we use maximum likelihood to assign labels to a scene. In the conventional way of doing so, object $o_{i}$ will be assigned label $l_{j}$ with probability $p_{i j}$, given by:

$$
p_{i j}=p\left(l_{j} \mid m_{i}\right) p\left(m_{i}\right)=p\left(m_{i} \mid l_{j}\right) p\left(l_{j}\right)
$$

where $m_{i}$ represents all the measurements we have made on object $o_{i}$, and $p\left(m_{i}\right)$ and $p\left(l_{j}\right)$ are the prior probabilities of measurements and labels, respectively. Probabilistic relaxation will update these probabilities according to the contextual information received from neighbouring regions. We do not follow that route here. Instead, we shall use the information coming from the other layers of knowledge to moderate this formula. Let us identify the units in the "verbs"

\footnotetext{
${ }^{1}$ The ideas of p-maps and m-maps first came to my knowledge by Robin Shirley of the Psychology Department of Surrey University, who passed away before he had the chance to make them more concrete and publish them.
} 
level of Fig. 1 by $f_{k}$, and the units at the descriptor level of Fig. 1 by $d_{l}$. Then we may choose label $l_{j_{i}}$ for object $o_{i}$ as follows:

$$
j_{i}=\arg \max _{j} \underbrace{\sum_{k} u_{j k} \sum_{l} v_{k l} c_{i l}}_{\text {utility_function }(i, j)} p_{i j}
$$

where $u_{j k}$ indicates how important is for an object with label $l_{j}$ to fulfil functionality $f_{k} ; v_{k l}$ indicates how important characteristic $d_{l}$ is for an object to have the possibility to fulfil functionality $f_{k}$, and $c_{i k}$ is the confidence we have that descriptor $d_{l}$ applies to object $o_{i}$.

Note that the value of the utility function expresses the evidence we have that region $o_{i}$ has the necessary characteristics to fulfil its role as object $l_{j}$. For example, if the label we consider of assigning to object $o_{i}$ is "balcony", the utility function must express whether this object has dimensions big enough to allow a human to stand on it, whether it is attached on a wall, and whether there is a door leading to it. All these are conditions that will allow an object to play the role of a balcony. A learning scheme must be able to learn the values of $u_{j k}$ and $v_{k l}$ either directly from examples (slowly and painfully), or by trusting its teacher, who having learnt those values himself, slowly and painfully over many years of human life experiences, directly inserts them to the computer learner. The computer learner then must have a tool box of processors of sensory inputs that will allow it to work out the values of $c_{i l}$.

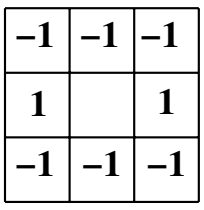

(a)

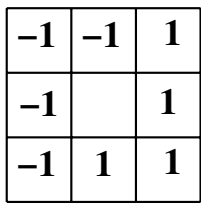

(b)

Fig. 2. (a) A local neighbourhood at the pixel level with globally consistent Markov parameters: if this field is relaxed it will lead to horizontal strips of similar labels which will be distinct from the labels above and below. In image processing it will lead to a texture pattern with strong horizontal directionality. (b) A local neighbourhood at the pixel level with globally inconsistent Markov parameters: the top left pixel tells the central pixel to be different from it; the central pixel, seen as the bottom right neighbour of the top left pixel, tells it to be similar to it.

\section{Conclusions}

I have argued here that learning is characterised by the ability to generalise, and that this can only be achieved if what is learnt is not the labels of the objects viewed, but the rules according to which these labels are assigned. I have also argued that this meta-knowledge may be transferred to the learner (the computer) 
directly by the teacher (the human developer), in the form of rules, or in the simplest way, by the human using the parameters of the algorithms according to their personal experience and intuition. This puts me at odds with the majority of the community of reviewers who tend to reject papers on the grounds that the parameters have been chosen ad hoc with no proper explanation: these are the cases of the teacher transplanting to the learner their painstakingly acquired knowledge. The alternative is for the learner each time to acquire this knowledge painfully slowly from thousands of examples.

I have also argued that we do not need globally consistent labellings of scenes. Global consistency will never allow us to label correctly the scene painted by Magritte of a train storming out of a fire place, because trains do not come out from fire places! It will never allow the computer to recognise green horses with 5 legs, but we, humans, do. So, what we need is fragments of reality and knowledge.

In computer vision, the idea of abandoning globally consistent solutions has now matured. This is not in isolation from other sciences. Strategy analysts talk about "fragments of learnt actions", and even mathematicians have long ago abandoned the idea of a globally self-consistent mathematical science: Bertrand Russell had to abandon the idea of globally consistent mathematics based on a small number of axioms, when Gödel's proof was published [15]. Natural systems are not globally consistent: they oscillate between states, and we, humans, manage to survive through this constantly dynamic, globally inconsistent and ambiguous world. A robotic system must be able to do the same and perhaps the only way to succeed in doing that is to be constructed so that it is content with a collection of fragments of understanding.

Acknowledgements. This work was supported by EU grant 027113 .

\section{References}

1. Cortes, C., Vapnik, V.N.: Support-Vector Networks. Machine Learning Journal 20, 273-297 (1995)

2. Christmas, W.J., Kittler, J., Petrou, M.: Structural matching in Computer Vision using Probabilistic Relaxation. IEEE Transactions on Pattern Analysis and Machine Intelligence 17, 749-764 (1995)

3. Devijver, P.A., Kittler, J.: On the edited nearest neighbour rule. In: Proc. 5th Int. Conf. on Pattern Recognition, pp. 72-80 (1980)

4. Guzman, A.: Computer Recognition of three-dimensional objects in a visual scene. Tech. Rep. MAC-TR-59, AI Laboratory, MIT (1968)

5. Heesch, D., Petrou, M.: Non-Gibbsian Markov Random Fields for object recognition. The British Machine Vision Conference (submitted, 2007)

6. Huffman, D.A.: Impossible Objects as Nonsense Sentences. Machine Intelligence 6, 295-323 (1971)

7. Hummel, R.A., Zucker, S.W.: One the foundations of relaxation labelling process. IEEE Transactions PAMI 5, 267-287 (1983)

8. Kindermann, R., Snell, J.L.: Markov Random Fields and their Applications. First book of the AMS soft-cover series in Contemporary Mathematics, American Mathematical Society (1980) 
9. Li, Z.: A neural model of contour integration in the primary visual cortex. Neural Computation 10, 903-940 (1998)

10. Li, Z.: Visual segmentation by contextual influences via intra-cortical interactions in the primary visual cortex. Networks:Computation in Neural Systems 10, 187-212

11. Li, Z.: Computational design and nonlinear dynamics of a recurrent network model of the primary visual cortex. Neural Computation 13, 1749-1780 (2001)

12. Lindley, D.V.: Making Decisions. John Wiley, Chichester (1985)

13. Marengoni, M.: Bayesian Networks and Utility Theory for the management of uncertainty and control of algorithms in vision systems. PhD thesis, University of Massachusetts (2002)

14. Miller, E.G., Matsakis, N.E., Viola, P.A.: Learning from one example through shared densities on transforms. In: CVPR (2000)

15. Nagel, E., Newman, J.R.: Gödel's Proof. Routledge and Kegan Paul (1959) ISBN: 0710070780

16. Pearl, J.: Probabilistic reasoning in intelligent systems: Networks of plausible inference. Morgan Kaufmann Publishers Inc., San Francisco (1988)

17. Petrou, M., Tabacchi, M., Piroddi, R.: Networks of ideas and concepts. IEEE Transactions of Man Machine and Cybernetics (submitted, 2007)

18. Petrou, M., Garcia Sevilla, P.: Image Processing, Dealing with Texture. Wiley, Chichester (2006)

19. Schlesinger, B.D., Hlavac, V.: Ten lectures on Statistical and Structural Pattern Recognition, ch. 10. Kluwer Academic Publishers, Dordrecht, The Netherlands (2002)

20. Stassopoulou, A., Petrou, M.: Obtaining the correspondence between Bayesian and Neural Networks. International Journal of Pattern Recognition and Artificial Intelligence 12, 901-920 (1998)

21. Stoddart, A.J., Petrou, M., Kittler, J.: On the foundations of Probabilistic Relaxation with product support. Journal of Mathematical Imaging and Vision 9, 29-48 (1998)

22. Tenenbaum, J.B., Griffiths, T.L., Kemp, C.: Theory-based Bayesian models of inductive learning and reasoning. Trends in Cognitive Sciences 10, 309-318 (2006)

23. Tong, S., Koller, D.: Support Vector Machine active learning with applications to text classification. Journal of Machine Learning Research 2, 45-66 (2001)

24. Walker, T.C., Miller, R.K.: Expert Systems Handbook: An Assessment of Technology and Applications. Prentice-Hall, Englewood Cliffs (1990)

25. Waltz, D.: Understanding line drawings of scenes with shadows. In: Winstone, P. (ed.) The Psychology of Computer Vision, pp. 19-91. McGraw-Hill, New York (1975), http://www.rci.rutgers.edu/ cfs/305_html/Gestalt/Waltz2.html

26. Winston, P.H.: Learning structural descriptions from examples. The psychology of computer vision, 157-209 (1975)

27. Zadeh, L.H.: A fuzzy algorithmic approach to the definition of complex or imprecise concepts. Int. J. Man-Machine Studies 8, 249-291 (1976)

28. http://www.emtech.net/learning-theories.htm 